\title{
Measurement of photodarkening in Yb-doped aluminosilicate fibers at elevated temperature
}

\author{
S. Yoo, A. J. Boyland, R. J. Standish, and J. K. Sahu
}

Photodarkening behavior in Yb-doped aluminosilicate fibers at elevated temperatures is reported. The fiber was core-pumped at $977 \mathrm{~nm}$ and the transmitted powers were monitored at 633 (probe) and $977 \mathrm{~nm}$ (pump) simultaneously with $\sim 47 \%$ of population inversion of $\mathrm{Yb}^{3+}$ ions. A saturated photodarkening loss was found, which is inversely proportional to temperature and, at $\sim 573 \mathrm{~K}$, the loss was negligible at the pump wavelength. From the decay curves at different temperatures, we found that the photodarkening involves second order kinetics to form color centers.

Introduction: Yb-doped fibers (YDF) exhibit excess loss covering $\mathrm{Yb}$ band when the fiber is irradiated at pump or shorter wavelength [1-2,]. The induced loss is proportional to the inversion level of the $\mathrm{Yb}^{3+}$ ions, [3] and also depends on host material [4-6]. Measurement of photodarkening, in most cases, relies on monitoring transmitted power of a visible probe beam located far away from the Yb-absorption band during pump irradiation [1, 6]. A loss in $\mathrm{Yb}$ band can be scaled from the loss at the probe wavelength due to similar spectral shape of the induced loss in a wide range of $\mathrm{Yb}$ fibers [1]. However, thermal treatment sometimes results in distortion in the loss spectrum, which invalidates this assumption [7]. On the other hand, the photodarkening measurement at high temperatures was proposed to accelerate the process in low $\mathrm{Yb}$ concentration fibers [8], but the measurement was performed only at visible wavelength and the temperature range was not very wide.

In this report, we present experimental results on photodarkening induced loss in Yb-doped aluminosilicate fibers at elevated temperatures. The induced loss was recorded at $633 \mathrm{~nm}$ (probe) and $977 \mathrm{~nm}$ (pump) simultaneously. The temperature dependence of the induced losses was related to kinetics of color center formation.

Experimental results: The photodarkening of the YDF was evaluated by monitoring the transmitted output power through the YDF under $977 \mathrm{~nm}$ irradiation. We used fiber-coupled and Bragg grating (FBG) stabilized $977 \mathrm{~nm}$ laser diode as a pump source. The output end of the pump fiber was spliced to the YDF and the throughput power was measured with a power meter. A He-Ne laser at $633 \mathrm{~nm}$ was used as a probe beam which coupled to the YDF and propagated the same direction as the pump beam. The probe beam was chopped and the output was detected by photodiode and lock-in amplifier. The measured output powers were recorded by data logger. We used $1 \mathrm{~cm}$ of the YDF to suppress amplified spontaneous emission. A uniform population inversion of $\mathrm{Yb}^{3+}$ around $47 \%$ was maintained throughout the YDF.

The tested YDF had 0.15 of core numerical aperture (NA) and $8.4 \mu \mathrm{m}$ /125 $\mu \mathrm{m}$ of core /cladding diameters. The Yb concentration was 17,000 ppm 
$w t \%$ in aluminosilicate host. The YDF was placed on a hot plate and covered by a metal plate to prevent possible heat loss from the top of the fiber.

Temperature was monitored with an external thermocouple placed by the YDF.

The temporal characteristics of the transmitted power at different temperatures are presented in Fig. 1. The initial power was scaled to unity for comparison. The decay curves below $573 \mathrm{~K}$ were well characterized with stretched exponential form given by:

$$
T(t)=A \exp \left(-\left(\frac{t}{\tau}\right)^{\beta}\right)+(1-A)
$$

where $\beta$ is a stretch parameter, $\tau^{-1}$ is a characteristic rate constant, and (1-A) is a saturated transmission. The fitting results are shown in Fig 1 as solid lines. The fitting parameters at different temperature are represented in the inset of Fig.1. We see that the transmitted powers quickly approach to the saturated value when temperature gets higher. In addition, the saturated transmission power is recorded larger with higher temperature, which is more pronounced at $633 \mathrm{~nm}$. Most interestingly, at $573 \mathrm{~K}$, the output power at $633 \mathrm{~nm}$ initially decays in $\sim 100 \mathrm{sec}$, but recovers gradually to its original value afterwards. This indicates effective thermal bleaching occurring even at the presence of strong population inversion, $\sim 47 \%$. Moreover, transmission at $977 \mathrm{~nm}$ showed negligible loss, with marginal initial drop.

The change in the inversion level due to the high temperatures possibly causes the different behaviours of the decay curves. However, the inversion levels at our temperature range are estimated nearly same with trivial variation of $\sim 1 \%$ at most, despite temperature effect on the spectroscopic properties of $\mathrm{Yb}^{3+}$ ions [9]. In addition, increase in the inversion level results in larger rate constant, but lower saturated transmission, i.e. higher loss [3]. Hence, the observed temperature dependence does not account for the change in the inversion level in our case.

We noticed that the ratio of saturated value at 633 and $977 \mathrm{~nm}$ is not preserved at different temperatures. For example, the ratio at $473 \mathrm{~K}$ exceeds $300 \%$ of that at $300 \mathrm{~K}$. Thus, monitoring at Yb-band is certainly necessary to offer explicit measure of the induced loss at the $\mathrm{Yb}$-band particularly when the fiber is exposed to various temperatures.

From the decay curves at different temperatures, we attempted to evaluate the kinetics involved in the photodarkening. Photodarkening is viewed as a color center formation process which involves: 1) releasing free carriers like electrons by activating precursors through photo-irradiation, and 2) forming colour centres by trapping the free electrons. [10] Hence, the number of the trapped electrons represents the photodarkening loss. If we assign the number of the free electrons as $n_{e}$, it obeys the rate equation:

$$
\frac{d n_{e}}{d t}=-k n_{e}^{m}
$$

where $k$ is a rate constant for the reaction and $m$ determines order of the reaction. The relationship between the rate constant and the temperature follows the Arrhenius type:

$$
k=A^{\prime} \exp \left(-E_{a} / k_{B} T\right)
$$


where $A^{\prime}$ is a temperature independent pre-exponential factor, $k_{B}$ is a Boltzmann constant, $T$ is temperature, and $E_{a}$ is an activation energy for the free electrons to overcome for the color center formation.

We used eq. (2) to fit the decay curves of $633 \mathrm{~nm}$ at different temperature to determine the rate constant, $k$, for each temperature. It was noticed that second order of the reaction, $m=2$, permitted a good fit, which are consistent with other photo-irradiation related color centers in silica fibers [11, 12]. The goodness of the fit was better than 0.995 with $m=2$, but no more than 0.963 with $m=1$. The rate constant at each temperature was then plotted as a function of the temperature to determine the activation energy. The activation energy was obtained as $0.07 \mathrm{eV}$ from the slope of the plot in Fig. 2. It should be noted that the obtained activation energy does not necessarily imply the barrier for thermal bleaching. However, it is interesting that the recovery was observed at the thermal energy of $3 / 2 k_{B} T=\sim 0.07 \mathrm{eV}$, or at $573 \mathrm{~K}$. We note that complete thermal bleaching of already darkened fibers demands higher temperature in a range of $670-900 \mathrm{~K}[5,7,13]$.

Conclusions: The photodarkening induced loss in Yb-doped aluminosilicate fibers at elevated temperatures was monitored at probe (633 nm) and pump $(977 \mathrm{~nm})$ wavelengths. The decay curves exhibited larger rate constant and saturated transmission at higher temperature. The photodarkening was accelerated at high temperatures but with higher saturated transmission. A complete recovery at $633 \mathrm{~nm}$ was observed at $573 \mathrm{~K}$ despite strong population inversion of $\sim 47 \%$. Furthermore, the induced loss at $\mathrm{Yb}$ band becomes negligible at $573 \mathrm{~K}$ during the irradiation.

The activation energy for the color center formation was calculated from the photodarkening decay curves. It was found to be $0.07 \mathrm{eV}$, which possibly explains the observed recovery in our fiber at $573 \mathrm{~K}$.

\section{ACKNOWLEDGMENTS}

This work has been supported in part by the Engineering and Physical Sciences Research Council UK (EPSRC) under EP/E033725/1 project.

\section{References}

1 Koponen, J. J., Söderlund, and H. J. Hoffman, : 'Measuring photodarkening from single-mode ytterbium doped silica fibers', Opt. Express, 2006, 14, (24), pp. 11539-11544.

2 Yoo, S., Basu, C., Boyland, A., Stone, C., Nilsson, J., Sahu, J. K., and Payne, D., : 'Photodarkening in Yb doped aluminosilicate fibers induced by 488nm irradiation', Opt. Lett., 2007, 32, (12), pp.1626-1629.

3 Koponen, J., Söderlund, M., Hoffman, H. J., Kliner, D. A. V., and Koplow, J. P. : 'Photodarkening measurements in large mode area fibers', Proc. SPIE, 2007, 6453, p. 64531E. 
4 Sahu, J. K., Yoo, S., Boyland, A. J., Basu, C., Kalita, M., Webb, A., Sones, C. L., Nilsson, J., and Payne, D. N. : '488 nm Irradiation Induced Photodarkening Study of Yb-Doped Aluminosilicate and Phosphosilicate Fibers', CLEO, San Jose, paper JTUA27, 2008.

5 Shubin, A. V., Yashkov, M. V., Melkumov, M. A., Smirnov, S. A., Bufetov, L. A., Dianov, E. M. : 'Photodarkening of alumosilicate and phosphosilicate Yb-doped fibers', CLEO EU, paper CJ3-1-THU, 2007.

6 Jetschke, S., Unger, S., Schwuchow, A., Leich, M., and Kirchhof, J. : 'Efficient $\mathrm{Yb}$ laser fibers with low photodarkening by optimization of the core composition', Opt. Express, 2008, 16, (20), pp. 15540-15545.

7 Söderlund, M., Ponsoda, J. J. M., Koplow, J. P., and Honkanen, S., : 'Heat-induced darkening and spectral broadening in photodarkened ytterbium-doped fiber under thermal cycling', Opt. Express, 2009, 17, (12), pp. 9940-9946.

8 Leich, M., Jetschke, S., Unger, S., and Reichel, V., : ‘Acceleration of photodarkening measurements in Yb-doped fibers by enhanced temperatures', CLEO EU, Munich, paper CE.P.29.MON, 2009.

9 Newell, T. C., Peterson, P., Gavrielides, A., and Sharma, M. P., : 'Temperature effects on the emission properties of Yb-doped optical fibers', Opt. Comm., 2007, 273, pp. 256-259.

10 Morasse, B., Cahtigny, S., Gagnon, É, Hovington, C., Martin, J-P., Sandro, J-P. : 'Low photodarkening single cladding ytterbium fibre amplifer', Proc. SPIE, 2007, 6453, p. 64530H.

11 Askins, C. G., and Putnam, M. A., : 'Photodarkening and photobleaching in fiber optic Bragg gartings', J. Lightwave Technol., 1997, 15, (8), pp. 1363-1370.

12 Henschel, H., and Baumann, E., : 'Effect of natural radioactivity on optical fibers of undersea cables', J. Lightwave Technol., 1996, 14, (5), pp. 724-731.

13 Jasapara, J., Andrejco, M., DiGiovanni, D., and Windeler, R. : 'Effect of heat and $\mathrm{H}_{2}$ gas on the photo-darkening of $\mathrm{Yb}^{3+}$ fibers', CLEO, Baltimore, paper CTUQ5, 2005.

\section{Authors' affiliations:}

S. Yoo, A. J. Boyland, R. J. Standish, and J. K. Sahu

(Optoelectronic Research Centre, University of Southampton, Highfield, Southampton SO17 1BJ, United Kingdom) 
E-mail address: sey@orc.soton.ac.uk

Fig. 1 Temporal characteristics of transmitted power at different temperatures at 633 and $977 \mathrm{~nm}$. The initial power was scaled to unity for comparison. Inset: Fitted rate constant, $\tau^{-1}$, and saturated transmission, (1-A), at different temperatures
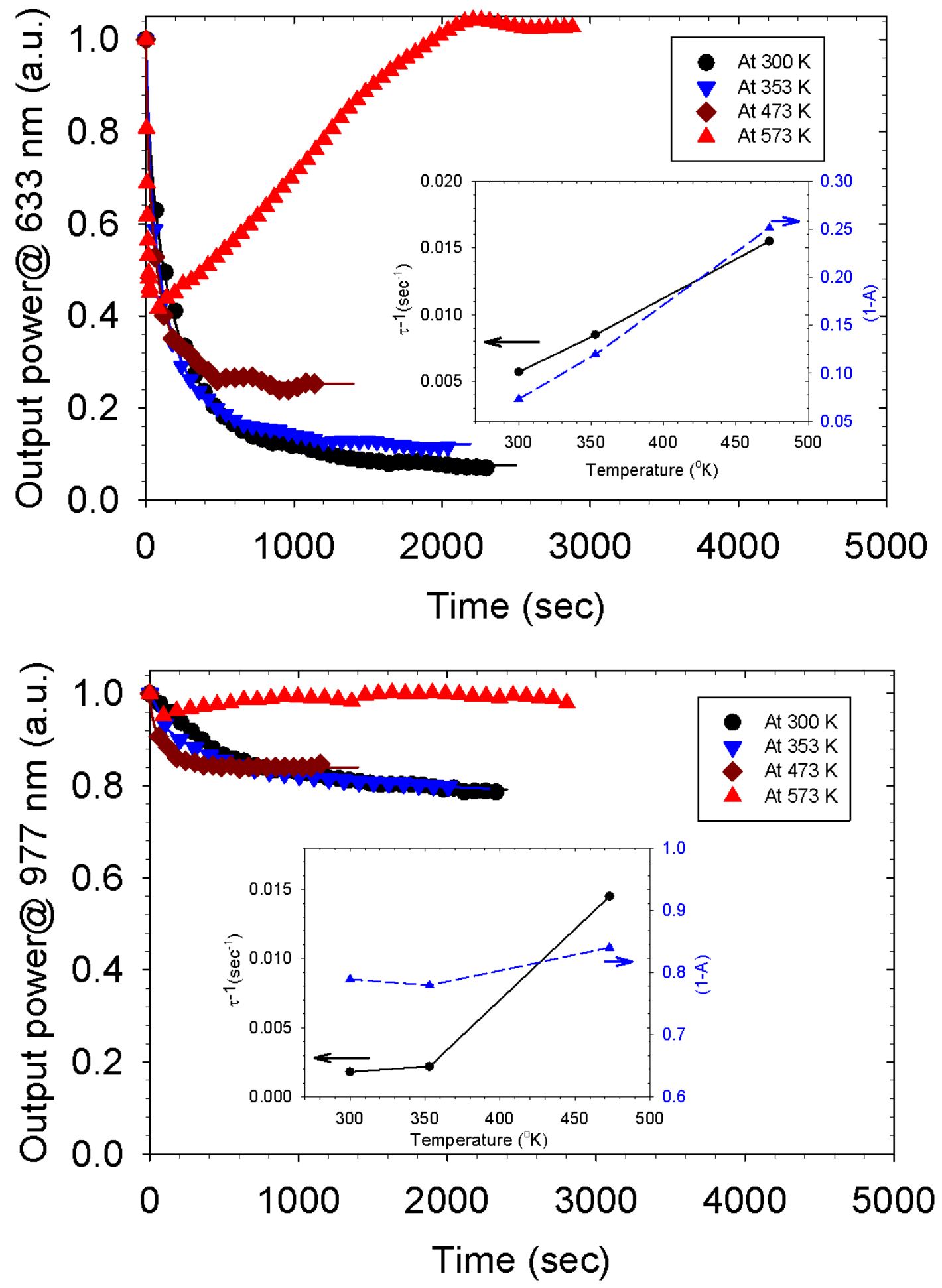
Fig. 2 Arrhenius plot of color center formation from free electrons

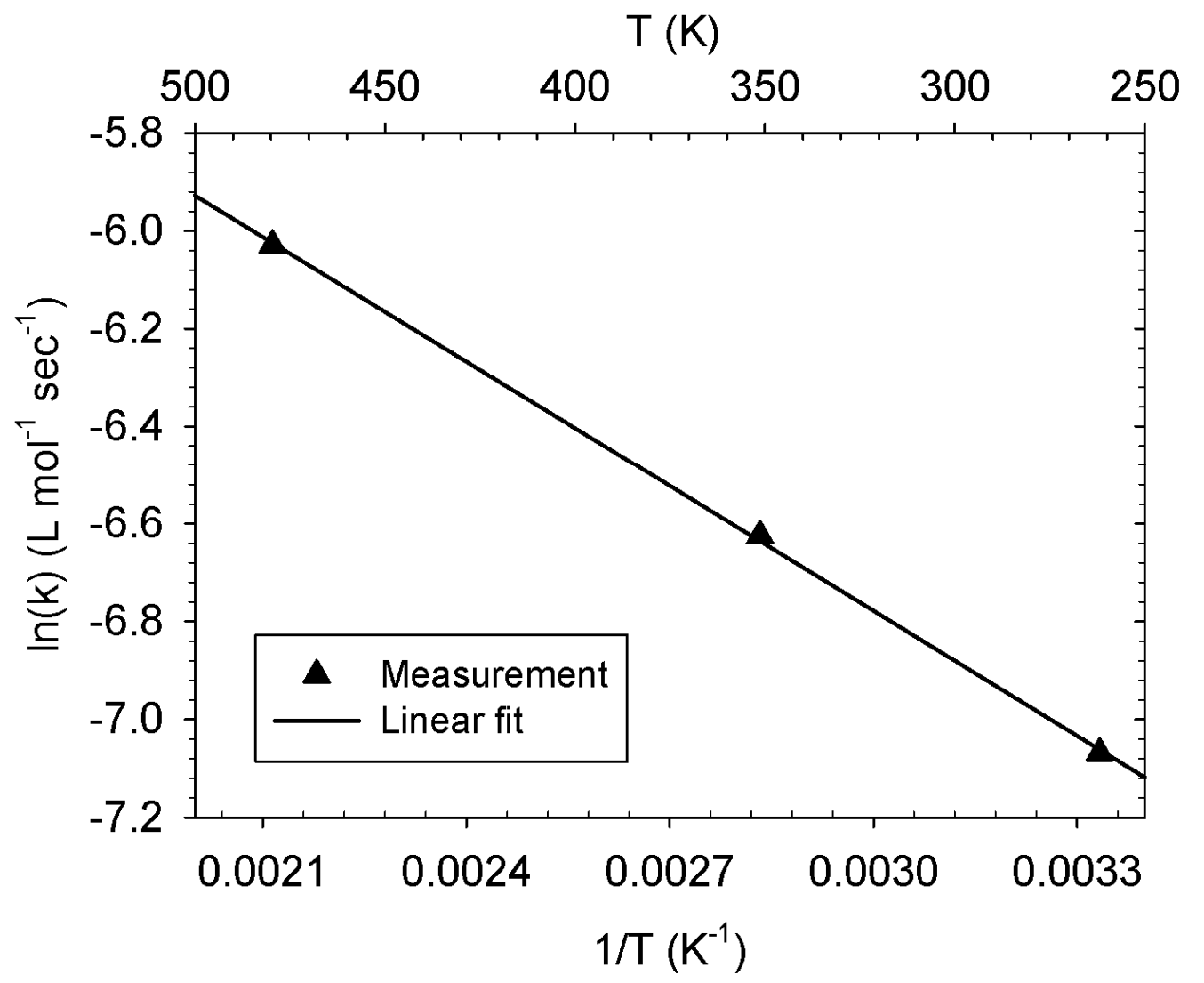

